\title{
Vascular Calcification and not Arrhythmia in Idiopathic Atrial Fibrillation Associates with Sex Differences in Diabetic Microvascular Injury miRNA Profiles
}

Citation for published version (APA):

Dudink, E., Florijn, B., Weijs, B., Duijs, J., Luermans, J., Peeters, F., Schurgers, L., Wildberger, J., Schotten, U., Bijkerk, R., Crijns, H. J., \& van Zonneveld, A. J. (2019). Vascular Calcification and not Arrhythmia in Idiopathic Atrial Fibrillation Associates with Sex Differences in Diabetic Microvascular Injury miRNA Profiles. MicroRNA (Shāriqah, United Arab Emirates), 8(2), 127-134.

https://doi.org/10.2174/2211536608666181122125208

Document status and date:

Published: 01/01/2019

DOI:

$10.2174 / 2211536608666181122125208$

Document Version:

Publisher's PDF, also known as Version of record

Document license:

Taverne

Please check the document version of this publication:

- A submitted manuscript is the version of the article upon submission and before peer-review. There can be important differences between the submitted version and the official published version of record.

People interested in the research are advised to contact the author for the final version of the publication, or visit the DOI to the publisher's website.

- The final author version and the galley proof are versions of the publication after peer review.

- The final published version features the final layout of the paper including the volume, issue and page numbers.

Link to publication

\footnotetext{
General rights rights.

- You may freely distribute the URL identifying the publication in the public portal. please follow below link for the End User Agreement:

www.umlib.nl/taverne-license

Take down policy

If you believe that this document breaches copyright please contact us at:

repository@maastrichtuniversity.nl

providing details and we will investigate your claim.
}

Copyright and moral rights for the publications made accessible in the public portal are retained by the authors and/or other copyright owners and it is a condition of accessing publications that users recognise and abide by the legal requirements associated with these

- Users may download and print one copy of any publication from the public portal for the purpose of private study or research.

- You may not further distribute the material or use it for any profit-making activity or commercial gain

If the publication is distributed under the terms of Article $25 \mathrm{fa}$ of the Dutch Copyright Act, indicated by the "Taverne" license above, 

tion Associates with Sex Differences in Diabetic Microvascular Injury miRNA Profiles

Elton Dudink ${ }^{\mathrm{a}, \#, *}$, Barend Florijn ${ }^{\mathrm{b}, \#}$, Bob Weijs ${ }^{\mathrm{a}}$, Jacques Duijs $^{\mathrm{b}}$, Justin Luermans $^{\mathrm{a}}$, Frederique Peeters ${ }^{\mathrm{a}}$, Leon Schurgers $^{\mathrm{c}}$, Joachim Wildberger ${ }^{\mathrm{d}}$, Ulrich Schotten ${ }^{\mathrm{e}}$, Roel Bijkerk ${ }^{\mathrm{b}}$, Harry J. Crijns ${ }^{\mathrm{a}, \#}$ and Anton Jan van Zonneveld ${ }^{\mathrm{b}, \#}$

${ }^{a}$ Department of Cardiology, Maastricht University Medical Center and Cardiovascular Research Institute Maastricht, P. Debyelaan 25, 6229 HX, Maastricht, The Netherlands; ${ }^{b}$ Department of Internal Medicine (Nephrology), Leiden University Medical Center and Einthoven Laboratory for Vascular and Regenerative Medicine, Albinusdreef 2, 2333 ZA, Leiden, The Netherlands; ${ }^{c}$ Department of Biochemistry,Maastricht University and Cardiovascular Research Institute Maastricht, Universiteitssingel 50, 6229 ER, Maastricht, The Netherlands; ${ }^{d}$ Department of Radiology and Nuclear Medicine, Maastricht University Medical Center and Cardiovascular Research Institute Maastricht, $P$. Debyelaan 25, 6229 HX, Maastricht, The Netherlands; ${ }^{e}$ Department of Physiology, Maastricht University and Cardiovascular Research Institute Maastricht, Universiteitssingel 50, 6229 ER, Maastricht, The Netherlands

\section{A R T I C L E H IS T O R Y}

Received: July 19, 2018

Revised: October 17, 2018

Accepted: November 16, 2018

DOI:

$10.2174 / 2211536608666181122125208$

CrossMark

\begin{abstract}
Background: Atrial Fibrillation (AF) in patients without concomitant cardiovascular pathophysiological disease, is called idiopathic Atrial Fibrillation (iAF). Nonetheless, iAF patients have often times subclinical coronary (micro) vascular dysfunction and, particularly in women, a higher prevalence of subsequent cardiovascular comorbidities. Previously, we identified a plasma miRNA association with diabetes and microvascular injury in Diabetic Nephropathy (DN) patients. Therefore, in this study we assessed whether plasma levels of these diabetic, microvascular injury associated miRNAs reflect microvascular integrity in iAF patients, associated with the presence of paroxysmal arrhythmia or instead are determined by concealed coronary artery disease.
\end{abstract}

Methods: Circulating levels of a pre-selected set of diabetic, (micro) vascular injury associated miRNAs, were measured in 59 iAF patients compared to 176 Sinus Rhythm (SR) controls. Furthermore, the presence of coronary artery and aortic calcification in each patient was assessed using Cardiac Computed Tomography Angiography (CCTA).

Results: Paroxysmal arrhythmia in iAF patients did not result in significant miRNA expression profile differences in iAF patients compared to SR controls. Nonetheless, coronary artery calcification (CAC) was associated with higher levels of miRNAs-103, -125a-5p, -221 and -223 in men. In women, CAC was associated with higher plasma levels of miRNA-27a and miRNA-126 and correlated with Agatston scores. Within the total population, ascending Aortic Calcification (AsAC) patients displayed increased plasma levels of miRNA-221, while women, in particular, demonstrated a Descending Aorta Calcification (DAC) associated increase in miRNA-212 levels.

Conclusions: Diabetic microvascular injury associated miRNAs in iAF are associated with subclinical coronary artery disease in a sex-specific way and confirm the notion that biological sex identifies iAF subgroups that may require dedicated clinical care.

Keywords: Atrial fibrillation, microRNA, sex-differences, vascular calcification, plasma, paroxysmal arrhythmia.

\section{INTRODUCTION}

There is a strong association between Atrial Fibrillation (AF) and vascular disease, as this arrhythmia usually occurs

*Address correspondence to this author at the Department of Cardiology, Maastricht University Medical Center and Cardiovascular Research Institute Maastricht, P. Debyelaan 25, 6229 HX, Maastricht, The Netherlands; Tel: +31 43387 5119; Fax: +31 43387 5104; E-mail: elton.dudink@mumc.nl ${ }^{\#}$ These authors contributed equally in patients with cardiovascular comorbidities [1,2] and, particularly in women upon diabetes mellitus [3]. However, in a subset of patients with AF, meticulous phenotyping cannot identify any concomitant cardiovascular disease, a condition called idiopathic AF (iAF). Mechanistic studies in iAF patients have identified several underlying pathophysiological mechanisms that pinpoint myocardial, microvascularperfusion abnormalities in iAF [4], including oxidative stress [5], systemic inflammation [6] and subclinical coronary artery 
disease [7]. Interestingly, in conjunction with female sex, these (micro) vascular comorbidities in iAF have been shown to augment the relative risk and event rates for stroke and transient ischemic attack [8]. Therefore, early monitoring of (micro) vascular integrity in iAF patients could aid to better characterize iAF subgroups that might benefit from dedicated treatment strategies.

MicroRNAs (miRNAs) are short non-coding RNA molecules that fine-tune gene expression and thereby control functionally-related biological pathways [9]. In human plasma, selected miRNAs circulate in levels that are different across (micro) vascular disease phenotypes [10] or biological sex [11]. Following this notion, we recently demonstrated that a subset of circulating miRNAs is associated with diabetic induced microvascular injury in Diabetic Nephropathy (DN) patients and correlated with conventional circulating markers of microvascular injury, such as angiopoietin-2 (Ang-2), soluble thrombomodulin (sTM) and Insulin-like Growth Factor (IGF) [12]. Previous miRNA expression profiling Studies in AF patients demonstrated that miRNA-126 [13] and miRNA-152 [14] levels are associated with prevalent arrhythmia, yet no relationship with vascular integrity or biological sex was established in these studies.

To determine whether circulating miRNAs could also serve as markers of (micro) vascular integrity in iAF, we assessed the association of plasma levels of 48 selected diabetic microvascular disease associated miRNAs which we previously found to be associated with (diabetic) conditions of (micro) vascular dysfunction [12, 15]. Next, because iAF has also been associated with increased subclinical coronary artery disease [7], we determined whether selected circulating miRNA levels were associated with coronary artery disease on Cardiac Computed Tomography Angiography (CCTA). Finally, because diabetes in women is a significant predictor of AF development [3] and women with iAF are at a higher risk of stroke or transient ischemic attack [8], we stratified circulating miRNA levels according to biological sex, to evaluate whether biological sex is a determinant of the association between vascular calcification and circulating miRNAs.

\section{METHODS}

\subsection{Patient Selection}

This study was approved by the Institutional Review Board and complied with the ethical principles of the Declaration of Helsinki. All patients gave written informed consent. Of all consecutive patients who underwent CCTA in the Maastricht University Medical Center between January 2008 and March 2011, 115 iAF patients referred for work-up for Pulmonary Vein Isolation (PVI) and 275 age, sex and PROCAM-score-matched [16] SR-controls were selected as previously described [7]. For RNA isolation we selected 235 patients (59 iAF, $176 \mathrm{SR}$ ) of whom EDTA-plasma was available.

\subsection{Computed Tomography}

Non-contrast enhanced coronary calcium scans were performed in all patients as described previously [7]. Scans were performed using a uniform scan protocol (240 to 400
$\mathrm{mA}$ (depending on body weight), at 80 to $120 \mathrm{kV}$ ) on a 64 slice CT platform (Brilliance, Philips Healthcare, Best, the Netherlands) and second generation dual source CT (Definition Flash, Siemens Healthcare, Forchheim, Germany). Cases and controls were equally distributed among both scanners. Two independent observers determined Calcification of the Coronary Arteries (CAC), ascending (AsAC) and Descending Aorta (DAC) using dedicated vendor specific software. The presence of calcification was defined as Agatston $>0$; the Agatston score was determined using a $3 \mathrm{~mm}$ CT slice thickness and a detection threshold of $\geq 130 \mathrm{HU}$ involving $\geq 1 \mathrm{~mm}^{2}$ area/lesion ( 3 pixels) [17]. In case of ambiguity, final judgment was reached in a consensus conference. Calcium above the origin of the right coronary artery to the end of scan range, or up to the origin of the brachiocephalic artery, was considered to be in the ascending aorta. Calcium present distal from the origin of the left subclavian artery up to the diaphragm was considered to be localized in the descending aorta.

\subsection{RNA Isolation and microRNA Profiling}

RNA was isolated from $200 \mu \mathrm{L}$ EDTA-plasma with 800 $\mu \mathrm{L}$ Trizol reagent (Invitrogen, Breda, the Netherlands) using the RNeasy Micro Kit (Qiagen, Venlo, the Netherlands) with an adapted protocol. Briefly, chloroform was added to the plasma/Trizol mixture and centrifuged for $15 \mathrm{~min}$ at $15000 \mathrm{~g}$. The aqueous phase was combined with 1.5 volumes of $100 \%$ ethanol, conveyed to a MinElute Spin column (Qiagen) and centrifuged for $15 \mathrm{~s}$ at $18000 \mathrm{~g}$. RNA was washed with 700 $\mu \mathrm{L}$ RWT buffer, twice with $500 \mu \mathrm{L}$ RPE buffer and centrifuged for $15 \mathrm{~s}$ at $18000 \mathrm{~g}$ after the first two washing steps and $2 \mathrm{~min}$ at $18000 \mathrm{~g}$ after the last washing step. RNA was eluted with $15 \mu \mathrm{L}$ RNase-free water.

MiRNAs were selected based on their previously established association with (micro)vascular dysfunction in profiling studies [12, 15]. (Supplementary Table 1) For miRNA cDNA synthesis, reverse transcription of total RNA was performed using the miRNA reverse transcription kit (Applied Biosystems, Foster City, CA). cDNA was pre-amplified using Megaplex PreAmp primers pools A V2.1 (Applied Biosystems) according to the manufacturer's protocol. Custom designed Megaplex cards were generated to determine the expression of the selected microRNAs. Megaplex arrays were run and analyzed on a $7900 \mathrm{HT}$ Fast Real-Time PCR System (Applied Biosystems). The obtained miRNA cycle threshold $(\mathrm{Ct})$-values were normalized using the median $\mathrm{Ct}$ value of the expression of 48 measured miRNAs per patient [18].

\subsection{Statistical Analysis}

Baseline characteristics are presented as mean \pm SD and differences between groups were compared using a student T-test, or number (\%) and compared using $\chi^{2}$-tests. MiRNAvalues are presented as mean $\pm \mathrm{SD}$ and the differences between the groups were assessed using a Student's T-test and general linear modeling to correct age, sex and vitamine $\mathrm{K}$ antagonist-use. In addition, miRNA-126 and miRNA-223 levels were corrected for aspirin use since aspirin inhibits platelet activation thereby decreasing the levels of both miRNAs [19]. 
Table 1. Clinical characteristics of patients with or without idiopathic atrial fibrillation, calcification in the coronary arteries, ascending- or descending aorta.

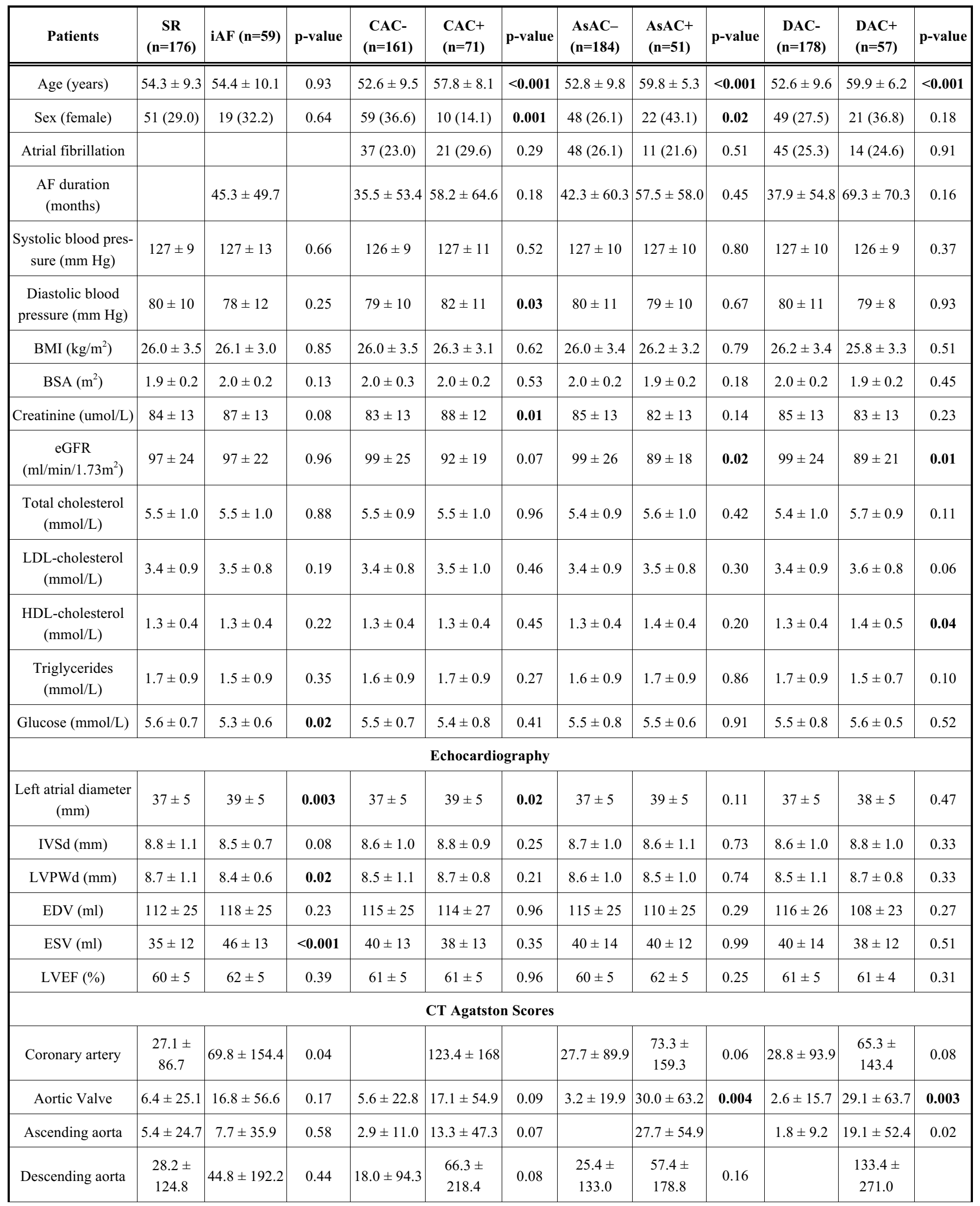




\begin{tabular}{|c|c|c|c|c|c|c|c|c|c|c|c|c|}
\hline Patients & $\begin{array}{c}\text { SR } \\
(n=176)\end{array}$ & iAF $(n=59)$ & p-value & $\begin{array}{l}\text { CAC- } \\
(n=161)\end{array}$ & $\begin{array}{l}\text { CAC+ } \\
(n=71)\end{array}$ & p-value & $\begin{array}{l}\text { AsAC- } \\
(n=184)\end{array}$ & $\begin{array}{l}\operatorname{AsAC}+ \\
(n=51)\end{array}$ & p-value & $\begin{array}{l}\text { DAC- } \\
(n=178)\end{array}$ & $\begin{array}{l}\text { DAC+ } \\
(n=57)\end{array}$ & p-value \\
\hline \multicolumn{13}{|c|}{ Medication } \\
\hline VKA & $0(0.0)$ & $15(25.4)$ & $<0.001$ & $6(3.7)$ & $9(12.7)$ & 0.01 & $12(6.5)$ & $3(5.9)$ & 0.87 & $9(5.1)$ & $6(10.5)$ & 0.14 \\
\hline Aspirin & $21(12.1)$ & $33(56.9)$ & $<0.001$ & $34(21.4)$ & $19(27.5)$ & 0.31 & $43(23.8)$ & $11(22.0)$ & 0.80 & $42(24.0)$ & $12(21.4)$ & 0.69 \\
\hline Beta blocker & $28(16.2)$ & $23(39.7)$ & $<0.001$ & $30(18.9)$ & $20(29.0)$ & 0.09 & $41(22.7)$ & $10(20.0)$ & 0.69 & $39(22.3)$ & $12(21.4)$ & 0.89 \\
\hline Digoxin & $0(0.0)$ & $8(13.8)$ & $<0.001$ & $6(3.8)$ & $2(2.9)$ & 0.74 & $5(2.8)$ & $3(6.0)$ & 0.27 & $5(2.9)$ & $3(5.4)$ & 0.37 \\
\hline $\begin{array}{c}\text { Nondihydropyridine } \\
\text { CCB }\end{array}$ & $1(0.6)$ & $5(8.6)$ & 0.001 & $1(0.6)$ & $5(7.2)$ & 0.004 & $6(3.3)$ & $0(0.0)$ & 0.19 & $3(1.7)$ & $3(5.4)$ & 0.14 \\
\hline Statin & $19(10.8)$ & 7 (11.9) & 0.82 & $17(10.6)$ & $9(12.7)$ & 0.64 & $17(9.2)$ & $9(17.6)$ & 0.09 & $20(11.2)$ & $6(10.5)$ & 0.88 \\
\hline
\end{tabular}

Data is expressed as mean \pm SD or $n(\%)$. AF, Atrial Fibrillation; SR, Sinus Rhythm; CAC, Coronary Artery Calcification; AsAC, Ascending Aorta Calcification; DAC, Descending Aorta Calcification; BMI, Body Mass Index; BSA, Body Surface Area; CAD, Coronary Artery Disease; VKA, Vitamin K Antagonist; CCB, Calcium Channel Blocker; ACE, Angiotensin Converting Enzyme; ARB, Angiotensin Receptor Blocker; IVSd, Interventricular Septum diameter; LVPWd, Left Ventricular Posterior Wall diameter; EDV, End Diastolic Volume; ESV, End Systolic Volume; LVEF, Left Ventricular Ejection Fraction.

Table 2. Differential expression of miRNAs in patients with or without calcification in the coronary arteries, ascending- or descending aorta.

\begin{tabular}{|c|c|c|c|c|c|c|c|c|c|}
\hline \multirow{2}{*}{$\begin{array}{l}\text { miRNA in } \\
\text { Patients }\end{array}$} & \multicolumn{3}{|c|}{ Total Population } & \multicolumn{3}{|c|}{ Female } & \multicolumn{3}{|c|}{ Male } \\
\hline & CAC- $(n=160)$ & $\mathrm{CAC}+(n=71)$ & p-value & CAC $-(n=58)$ & $\mathrm{CAC}+(n=10)$ & p-value & CAC $-(n=100)$ & $\mathrm{CAC}+(n=61)$ & p-value \\
\hline miRNA-103 & $0.36 \pm 0.32$ & $0.50 \pm 0.36$ & 0.013 & $0.30 \pm 0.28$ & $0.49 \pm 0.51$ & 0.130 & $0.39 \pm 0.33$ & $0.50 \pm 0.34$ & 0.150 \\
\hline miRNA-126\# & $26.78 \pm 13.76$ & $30.07 \pm 13.70$ & 0.05 & $25.83 \pm 12.15$ & $36.47 \pm 15.38$ & 0.012 & $27.33 \pm 14.64$ & $29.04 \pm 13.14$ & 0.341 \\
\hline miRNA-221 & $6.66 \pm 6.54$ & $9.03 \pm 7.56$ & 0.038 & $6.23 \pm 8.68$ & $7.69 \pm 4.03$ & 0.782 & $6.91 \pm 4.98$ & $9.25 \pm 7.99$ & 0.05 \\
\hline \multirow[t]{2}{*}{ miRNA-223\# } & $397.05 \pm 229.29$ & $472.14 \pm 215.75$ & 0.035 & $415.69 \pm 227.16$ & $515.57 \pm 256.38$ & 0.103 & $390.54 \pm 231.53$ & $465.02 \pm 209.96$ & 0.043 \\
\hline & AsAC- $(n=184)$ & $\operatorname{AsAC}+(n=51)$ & & AsAC- $(n=48)$ & AsAC $+(n=22)$ & & AsAC- $(n=136)$ & AsAC $+(n=29)$ & \\
\hline miRNA-212 & $0.039 \pm 0.039$ & $0.034 \pm 0.020$ & 0.62 & $0.028 \pm 0.016$ & $0.041 \pm 0.027$ & 0.035 & $0.042 \pm 0.045$ & $0.034 \pm 0.019$ & 0.346 \\
\hline
\end{tabular}

Comparisons within the total population are adjusted for age, sex, and vitamin-K antagonists, while female and male group comparisons are adjusted for age and the use of vitamin $\mathrm{K}$ antagonists. MiRNA expression values marked with\# are corrected for aspirin use. MiRNA relative expression data are presented as mean $\pm \mathrm{SD}$.

\section{RESULTS}

\subsection{Baseline Characteristics of iAF Patients Compared to SR Controls}

Baseline characteristics of the iAF patients and the SR controls are listed in Table 1. Patients with iAF had a significantly larger left atrial diameter (LAd) and end systolic volume (ESV), while the left ventricular posterior wall diameter (LVPWd) was significantly smaller. Significantly different CAC, AsAC and DAC Agatston scores between groups are listed in Table 1. Patients with CAC were average older, more often male and had larger LAds while patients with or without aortic calcification were comparable except for their age.

\subsection{Coronary Artery Calcification and not Arrhytmia Associates with Sex-Specific Circulating miRNA Profiles in iAF}

To investigate whether the presence of paroxysmal arrhythmia in iAF patients associates with circulating levels of the selected panel of 48 miRNAs, we assessed their plasma levels in iAF patients compared to SR controls (Table 2). After correction for biological sex and age, no plasma miRNAs were found to be associated with a history of AF in the total iAF patient population. Next, to determine the contribution of subclinical-CV comorbidities on circulating miRNA expression, we assessed the association of CAC with plasma miRNAs. In the total population, CAC associated with significantly higher levels of miRNAs-103, 125a-5p, 221 and 


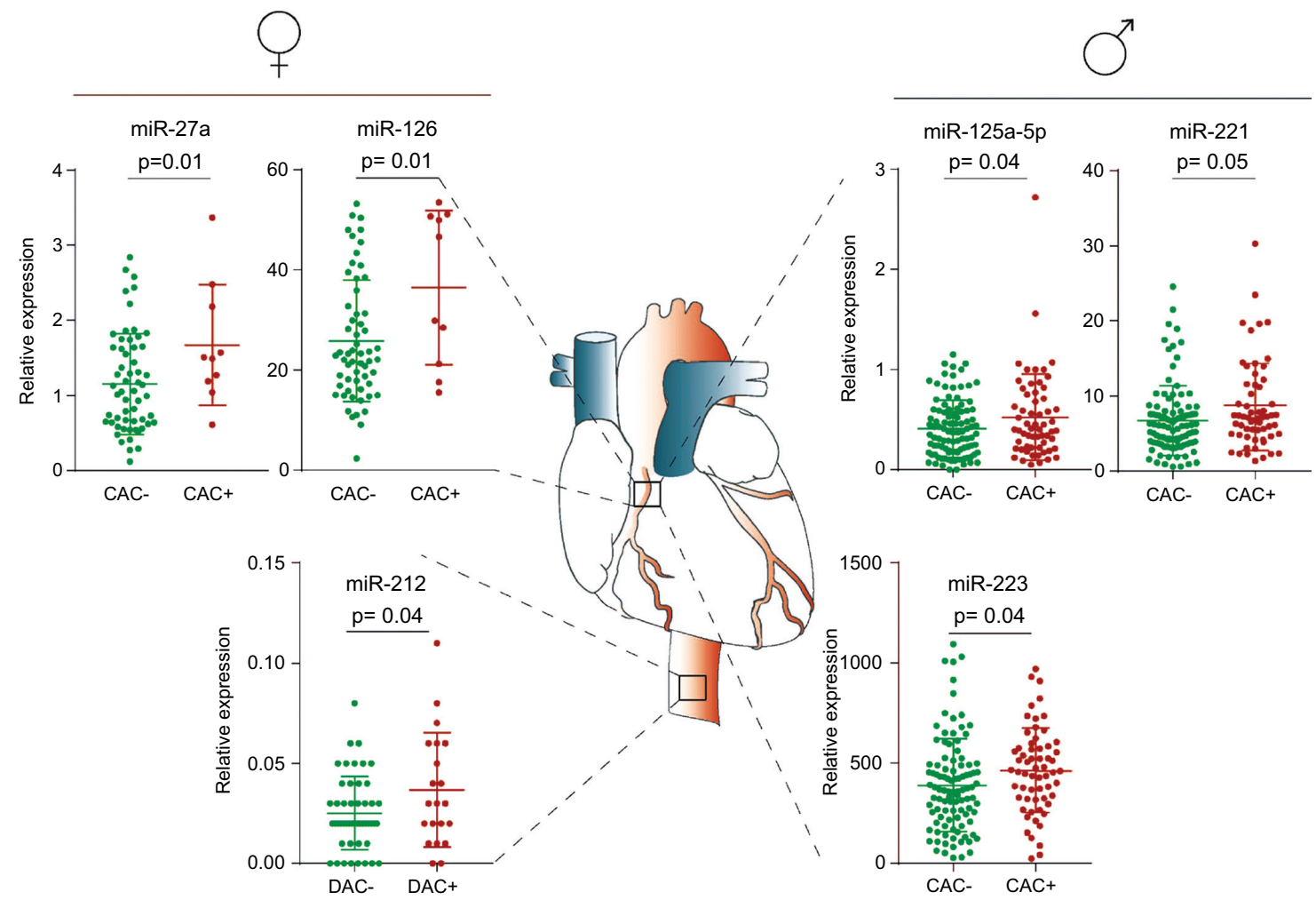

Fig. (1). Differential circulating miRNAs in women and men either with or without Coronary Artery Calcification (CAC), Ascending Aorta Calcification (AsAC) and Descending Aorta Calcification (DAC). Comparisons are adjusted for age, aspirin and Vitamin $\mathrm{K}$ antagonist use. Only significantly differently expressed miRNAs are shown.

223, of which the latter three were increased in in men only (Table 2, Fig. 1). In contrast, analysis of CAC associated miRNAs in women revealed a significantly increased expression of miRNA-27a and miRNA-126.

\subsection{Circulating Levels of miRNA-221and miRNA-212 Levels in Women, Associate with Aortic Calcification}

Next, following the identification of coronary calcification associated miRNAs, we examined the relationship between specific miRNAs and aorta calcification. Within the total patient population, the presence of AsAC resulted in lower levels of miRNA-221. Interestingly, upon stratification for biological sex, the AsAC association with miRNA-221 still displayed a trend towards lower levels, although these differences were not significant for both men and women. However, the presence of DAC in women but not in men was associated with higher expression levels of miRNA-212 expression in women.

\section{DISCUSSION}

This study shows that in iAF, instead of arrhythmia, subclinical CV comorbidities such as CAC, AsAC and DAC, are associated with plasma levels of a selected set of miRNAs. Interestingly, when $\mathrm{CAC}$, AsAC and DAC group comparisons were stratified for biological sex we observed a sexspecific, significant increase in miRNA-27a and miRNA-126 levels in women with a positive CAC score, while the levels of miRNA-125a-5p, miRNA-221 and miRNA-223 were higher in CAC positive men. Furthermore, circulating levels of miRNA-221 were elevated in patients with ascending aor- ta calcification, while miRNA-212 expression was associated with descending aorta calcification in women only.

Several of the differentially expressed miRNAs in this study have already shown promising potential as biomarker for clinical diagnostics and patients' response to treatment. As such, we previously demonstrated that the plasma levels of miRNA-126 increase, while miRNA-223 levels decrease in DN patients and normalize to healthy control levels, upon a simultaneous pancreas kidney (SPK) transplantation [12]. Furthermore, in type 2 diabetes patients, both miRNA-126 and -223 display a positive response to drug treatment [20] while miRNA-126 was found to predict platelet activation and the response to aspirin therapy [21] Also, miRNA-223 can be used in clinical care because its elevated levels were prognostic for cardiovascular mortality in patients with coronary artery disease (4). Interestingly, plasma levels of miRNA-27a were similarly found to provide additional aid in dedicated patient care because a reduction in its circulating levels was associated with rehospitalization for heart failure patients (5). Therefore, the observed association of these miRNAs with vascular calcification in this study could potentially indicate a pathophysiology of subclinical coronary (micro) vascular dysfunction in iAF patients. Nonetheless, more miRNA expression profiling and functional studies in iAF patients are warranted to validate this relationship.

Studies on the relationship between iAF and circulating miRNAs are limited, although several studies reported changes in circulatory miRNAs in AF. In particular, miRNA-150 has consistently been associated with AF, and was repeatedly found to be decreased in (older) patients with AF 
[22-24]. We could not confirm this association in the present study with iAF patients. It could be argued that the lowering of miRNA-150 levels in AF are mostly related to the contribution of $\mathrm{CV}$ comorbidities, particularly because miRNA150 is a marker of advanced atherosclerosis [25]. Therefore, next to its association with arrhythmia in AF, this miRNA150 change could also be related to the simultaneous prevalence of hypertension [22], heart failure [23] or diabetes [24] in these patient cohorts. Likewise, a similar relationship was identified between AF and plasma levels of miRNA-21 [24], a marker of cardiac fibrosis [26] and miRNA-152 [14] which is decreased in high glucose conditions [27]. Again these miRNAs levels were also not altered in our iAF patients. A possible explanation for this discrepancy could be that our patients were not diabetic and relatively young, whereas previous miRNA-21 expression changes were predominantly observed in older patients with a more severe AF phenotype, and an ensuing state of atrial ischemia and fibrosis [26]. Taken together, it could be argued that the previously identified circulatory miRNA profiles in AF are predominantly determined by an ensuing presence of arrhythmia in conjunction with severe CV comorbidities that are absent or less manifest in our iAF patient cohort.

The observed increase of the identified circulating miRNAs in this study could be explained by looking at their functional role within the vasculature system. For instance, in the setting of atherosclerosis, increased miRNA-103 expression in activated endothelial cells (ECs) represses endothelial KLF4 expression and thereby cellular quiescence [28] whereas an increased endothelial miRNA-212 expression is anti-angiogenic [29]. As such, the higher levels of miRNA103 and miRNA-212 in this study may suggest a pathophysiology of endothelial dysfunction and inflammation. Furthermore, although miRNA-126 normally favours vascular regeneration [30], the association of increased levels of miRNA-126 with vascular calcification in this study could indicate that, although speculative, more plasma miRNA-126 reflects a vascular regenerative feedback response in these iAF patients. Moreover, miRNAs are distributed among plasma miRNA carriers, such as exosomes [31], circulating high density lipoprotein (HDL) [32] or Argonaute-2 (Ago2) [33]. These plasma miRNA carriers enable the functional delivery of miRNAs at distal sites to pass on a miRNA mediated feedback loop in tissue inflammation, repair and regeneration. The observed increased expression of plasma miRNA-126, $-27 \mathrm{a},-125 \mathrm{a}-5 \mathrm{p},-212$ and -223 could have such carrier dependent impact. For instance, CD34+ peripheral blood mononuclear cells (PBMCs) derived exosomal miRNA-126 was found to be proangiogenic [34] whereas HDLmiRNA-223 transfer repressed endothelial activation and inflammation [35]. Taken together, it could be argued that the observed increase of plasma miRNAs may reflect underlying pathophysiology or a regenerative response.

Diabetes mellitus is a highly significant predictor of AF in women [3]. Furthermore, in iAF patients the relative risk of stroke, Transient Ischemic Attack (TIA) and heart failure are at least doubled in woman compared to man [8] further emphasizing the action of sex-specific mechanisms in cardiovascular pathophysiology. These mechanisms may also involve the action of sex-specific miRNAs. In woman, miRNA expression levels can differ from those in man due to oestrogen-dependent production or as a consequence of a gene-dose effect following incomplete X-chromosome inactivation [36]. This could be possible because the Xchromosome in humans and mice is relatively enriched for miRNAs (118 annotated miRNAs on the human Xchromosome, source: miRBase vs. 22) with a two-fold higher miRNA density compared to autosomes. These $\mathrm{X}$ chromosome-located miRNAs (X-linked miRNAs) could potentially display an increased expression in endothelial cells (ECs) and vascular smooth muscle cells (VSMCs) from the cardiovascular system due to incomplete $\mathrm{X}$-chromosome inactivation (XCI) [11]. This could not explain that the $\mathrm{X}$ linked miRNA-221 and miRNA-223 in this study were increased in male patients only. Therefore, another explanation for their sex biased miRNA expression could be the simultaneous expression with a sex-biased host gene or the sex specific expression of components of the miRNA processing machinery, such as Dicer or Drosha [11]. Therefore, another interesting mechanism for sexual dimorphism of miRNA expression could be the preferential binding of certain transcription factors (TFs) to the proximal genomic regions of particular sex-biased miRNA genes (sex-biased miRNAs have $15.7 \mathrm{TF}$ binding sites in vicinity compared to $9.1 \mathrm{TF}$ binding sites for non-biased miRNAs) [37]. Since, so far, 33 female specific miRNA-regulating TFs have been identified compared to 56 male specific miRNA regulating TFs [37], it increases the likelihood that this regulatory control will develop differently between men and women.

In this study, women with coronary artery- and aortic calcification displayed higher circulating levels of miRNA27a, miRNA-212 and miRNA-126 compared to men. Since women display higher levels of miRNA-126 during the ovulatory phase, and oestrogen was demonstrated to increase miRNA-126 expression to control the inflammatory response of ECs, a role for oestrogen in transcription of this vascularprotective miRNA in CAC positive women can be proposed [38]. Furthermore, the observed female-related difference in miRNA-27a expression is consistent with observations that this miRNA is decreased in women with gestational diabetes [39] but increased in women with polycystic ovary syndrome [40], suggesting that miRNA-27a is responsive to a synchronous state of high estrogen and metabolic dysfunction in women. Moreover, in contrast to the studied women in this cohort, CAC positive men displayed significantly higher levels of miRNA-221 and miRNA-223. Interestingly, miRNA-221, is known as a promoter of vascular calcification [41] and regulated by androgen hormone levels [42], while miRNA-223 is a known biomarker of CAC. [43] Therefore, although speculative, the male specific expression of these miRNAs in this cohort could be related to a malespecific cause or outcome of $\mathrm{CV}$ comorbidities in iAF patients. Taken together, it could be argued that the association of these sex-specific circulating miRNAs with vascular calcification in IAF patients confirms the notion that biological sex should be taken into account when deciding on vascular prophylactic therapy, including anticoagulation $[8,44]$.

This miRNA profiling study in iAF patients has several limitations. Measured miRNAs were selected based on their previous association with vascular injury. However, since numerous miRNAs are (mechanistically) involved in $\mathrm{CV}$ disease, we cannot exclude more miRNA changes in iAF 
than those observed in this study. Furthermore, this study was not followed by miRNA expression validation studies in independent $\mathrm{iAF}$ cohorts nor did we conduct intervention studies to evaluate whether the identified miRNAs are the cause or the outcome of (vascular calcification in) iAF. Therefore, future studies to validate these circulating levels of miRNAs in larger iAF patient cohorts are needed. However, since vascular calcification and age in iAF patients both augment coronary calcification [45] and because platelet activation hampers the biomarker potential of miRNA126 and miRNA-223 [19], a strength of this study is the statistical adjustment of differentially regulated miRNAs for the use of aspirin and vitamin $\mathrm{K}$ antagonists.

\section{CONCLUSION}

Circulating miRNAs in iAF do not associate with the mere presence of paroxysmal arrhythmia but instead display a sex-specific association with subclinical vascular calcification. This sex specific miRNA profile association with vascular calcification in iAF patients, confirms the existence of sex specific etiologies of vascular disease, and suggests that sex specific strategies for the prevention of vascular complications should be employed.

\section{ETHICS APPROVAL AND CONSENT TO PARTICI- PATE} Board.

This study was approved by the Institutional Review

\section{HUMAN AND ANIMAL RIGHTS}

No animal were used in the study. The reported experiments in accordance with the ethical standards of the committee responsible for human experimentation (institutional and national), and with the Helsinki Declaration of 1975, as revised in 2008 (http://www.wma.net/en/20activities/10 ethics/10helsinki/).

\section{CONSENT FOR PUBLICATION}

All patients gave written informed consent.

\section{CONFLICT OF INTEREST}

One author Joachim E. Wildberger of this manuscript declares institutional relationships with the following companies: Siemens AG, Bayer Healthcare, Philips, GE, all outside submitted work.

All other authors report no relationships that could be construed as a conflict of interest.

\section{ACKNOWLEDGEMENTS}

We acknowledge the support from the Netherlands Cardiovascular Research Initiative: an initiative with support of the Dutch Heart Foundation, CVON 2014-9: Reappraisal of Atrial Fibrillation: interaction between hyper Coagulability, Electrical remodeling, and Vascular destabilization in the progression of AF (RACE V). Furthermore, this work was partly supported by a grant from the Dutch Heart Foundation, Queen of Hearts: Improving diagnosis of CVD in women, (2013T084). Funders did not have any role in the design and conduct of the study.

\section{SUPPLEMENTARY MATERIAL}

Supplementary material is available on the publisher's website along with the published article.

\section{REFERENCES}

[1] Nieuwlaat R, Prins MH, Le Heuzey JY, et al. Prognosis, disease progression, and treatment of atrial fibrillation patients during 1 year: follow-up of the Euro heart survey on atrial fibrillation. Eur Heart J 2008; 29(9): 1181-9.

[2] Linz D, Elliott AD, Marwick TH, Sanders P. Biomarkers and newonset atrial fibrillation to assess atrial cardiomyopathy. Int J Cardiol 2017; 248: 208-10.

[3] Nichols GA, Reinier K, Chugh SS. Independent contribution of diabetes to increased prevalence and incidence of atrial fibrillation. Diabetes Care 2009; 32(10): 1851-6.

[4] Skalidis EI, Hamilos MI, Karalis IK, Chlouverakis G, Kochiadakis GE, Vardas PE. Isolated atrial microvascular dysfunction in patients with lone recurrent atrial fibrillation. J Am Coll Cardiol 2008; 51(21): 2053-7.

[5] Mihm MJ, Yu F, Carnes CA, et al. Impaired myofibrillar energetics and oxidative injury during human atrial fibrillation. Circulation 2001; 104(2): 174-80.

[6] Smit MD, Maass AH, De Jong AM, et al. Role of inflammation in early atrial fibrillation recurrence. Europace 2012; 14(6): 810-7.

[7] Weijs B, Pisters R, Haest RJ, et al. Patients originally diagnosed with idiopathic atrial fibrillation more often suffer from insidious coronary artery disease compared to healthy sinus rhythm controls. Heart Rhythm 2012; 9(12): 1923-9.

[8] Andersson T, Magnuson A, Bryngelsson IL, et al. Gender-related differences in risk of cardiovascular morbidity and all-cause mortality in patients hospitalized with incident atrial fibrillation without concomitant diseases: a nationwide cohort study of 9519 patients. Int J Cardiol 2014; 177(1): 91-9.

[9] van Zonneveld AJ, Rabelink TJ, Bijkerk R. MiRNA-coordinated networks as promising therapeutic targets for acute kidney injury. Am J Pathol 2017; 187(1): 20-4.

[10] Creemers EE, Tijsen AJ, Pinto YM. Circulating microRNAs: novel biomarkers and extracellular communicators in cardiovascular disease? Circ Res 2012; 110(3): 483-95.

[11] Florijn BW, Bijkerk R, van der Veer EP, van Zonneveld AJ. Gender and cardiovascular disease: are sex-biased microRNA networks a driving force behind heart failure with preserved ejection fraction in women? Cardiovasc Res 2018; 114(2): 210-25.

[12] Bijkerk R, Duijs JM, Khairoun M, et al. Circulating microRNAs associate with diabetic nephropathy and systemic microvascular damage and normalize after simultaneous pancreas-kidney transplantation. Am J Transplant 2015; 15(4): 1081-90.

[13] Wei XJ, Han M, Yang FY, et al. Biological significance of mir-126 expression in atrial fibrillation and heart failure. Braz J Med Biol Res 2015; 48(11): 983-9.

[14] McManus DD, Lin H, Tanriverdi K, et al. Relations between Circulating microRNAs and atrial fibrillation: data from the framingham offspring study. Heart Rhythm 2014; 11(4): 663-9.

[15] Bijkerk R, Florijn BW, Khairoun M, et al. Acute rejection after kidney transplantation associates with circulating microRNAs and vascular injury. Transplant Direct 2017; 3(7): e174.

[16] Assmann G, Cullen P, Schulte H. Simple scoring scheme for calculating the risk of acute coronary events based on the 10-year follow-up of the prospective cardiovascular munster (procam) study. Circulation 2002; 105(3): 310-5.

[17] Agatston AS, Janowitz WR, Hildner FJ, Zusmer NR, Viamonte M, Jr., Detrano R. Quantification of coronary artery calcium using ultrafast computed tomography. J Am Coll Cardiol 1990; 15(4): 82732 .

[18] Mestdagh P, Van Vlierberghe P, De Weer A, et al. A novel and universal method for microRNA Rt-Qper data normalization. Genome Biol 2009; 10(6): R64.

[19] Khairoun M, de Koning EJ, van den Berg BM, et al. Microvascular damage in type 1 diabetic patients is reversed in the first year after simultaneous pancreas-kidney transplantation. Am J Transplant 2013; 13(5): 1272-81. 
[20] Catanzaro G, Besharat ZM, Chiacchiarini M, et al. Circulating microRNAs in elderly type 2 diabetic patients. Int $\mathrm{J}$ Endocrinol 2018; 2018: 6872635 .

[21] de Boer HC, van Solingen C, Prins J, et al. Aspirin treatment hampers the use of plasma microRNA-126 as a biomarker for the progression of vascular disease. Eur Heart J 2013; 34(44): 3451-7.

[22] Liu Z, Zhou C, Liu Y, et al. The expression levels of plasma micoRNAs in atrial fibrillation patients. PLoS One 2012; 7(9): e44906.

[23] Goren Y, Meiri E, Hogan C, et al. Relation of reduced expression of miR-150 in platelets to atrial fibrillation in patients with chronic systolic heart failure. Am J Cardiol 2014; 113(6): 976-81.

[24] McManus DD, Tanriverdi K, Lin H, et al. Plasma microRNAs are associated with atrial fibrillation and change after catheter ablation (the mirhythm study). Heart Rhythm 2015; 12(1): 3-10.

[25] Karakas M, Schulte C, Appelbaum S, et al. Circulating microRNAs strongly predict cardiovascular death in patients with coronary artery disease-results from the large atherogene study. Eur Heart $\mathbf{J}$ 2017; 38(7): 516-23.

[26] Tao H, Zhang M, Yang JJ, Shi KH. MicroRNA-21 via dysregulation of ww domain-containing protein 1 regulate atrial fibrosis in atrial fibrillation. Heart Lung Circ 2018; 27(1): 104-13.

[27] Haque R, Hur EH, Farrell AN, Iuvone PM, Howell JC. Microrna152 represses VEGF and tgfbetal expressions through posttranscriptional inhibition of (pro)renin receptor in human retinal endothelial cells. Mol Vis 2015; 21: 224-35.

[28] Hartmann P, Zhou Z, Natarelli L, et al. endothelial dicer promotes atherosclerosis and vascular inflammation by miRNA-103mediated suppression of Klf4. Nat Commun 2016; 7: 10521.

[29] Kumarswamy R, Volkmann I, Beermann J, et al. Vascular importance of the miR-212/132 cluster. Eur Heart J 2014; 35(45): 3224-31.

[30] Bijkerk R, van Solingen C, de Boer HC, et al. Hematopoietic microRNA-126 protects against renal ischemia/reperfusion injury by promoting vascular integrity. J Am Soc Nephrol 2014; 25(8): 171022.

[31] Valadi H, Ekstrom K, Bossios A, Sjostrand M, Lee JJ, Lotvall JO. Exosome-mediated transfer of mRNAs and microRNAs is a novel mechanism of genetic exchange between cells. Nat Cell Biol 2007; 9(6): 654-9.

[32] Vickers KC, Palmisano BT, Shoucri BM, Shamburek RD, Remaley AT. microRNAs are transported in plasma and delivered to recipient cells by high-density lipoproteins. Nat Cell Biol 2011; 13(4): 423-33.
[33] Arroyo JD, Chevillet JR, Kroh EM, et al. Argonaute2 complexes carry a population of circulating microRNAs independent of vesicles in human plasma. Proc Natl Acad Sci U S A 2011; 108(12): 5003-8.

[34] Mocharla P, Briand S, Giannotti G, et al. Angiomir-126 expression and secretion from circulating CD34(+) and Cd14(+) Pbmcs: role for proangiogenic effects and alterations in type 2 diabetics. Blood 2013; 121(1): 226-36.

[35] Tabet F, Vickers KC, Cuesta Torres LF, et al. HDL-Transferred microRNA-223 regulates Icam-1 expression in endothelial cells. Nat Commun 2014; 5: 3292.

[36] Florijn BW, Bijkerk R, van der Veer E, van Zonneveld AJ. Gender and cardiovascular disease: are sex-biased miRNA networks a driving force behind heart failure with preserved ejection fraction in women? Cardiovasc Res 2018; 114(2): 210-25.

[37] Cui C, Yang W, Shi J, et al. Identification and analysis of human sex-biased microRNAs. Genomics Proteomics Bioinfo 2018; 16(3): 200-11.

[38] Li P, Wei J, Li X, et al. 17beta-estradiol enhances vascular endothelial ets-1/miR-126-3p expression: the possible mechanism for attenuation of atherosclerosis. J Clin Endocrinol Metab 2017; 102(2): 594-603

[39] Ameling S, Kacprowski T, Chilukoti RK, al. Associations of circulating plasma microRNAs with age, body mass index and sex in a population-based study. BMC Med Genomics 2015; 8: 61.

[40] Wang M, Sun J, Xu B, et al. Functional characterization of microRNA-27a-3p expression in human polycystic ovary syndrome. Endocrinology 2018; 159(1): 297-309.

[41] Mackenzie NC, Staines KA, Zhu D, Genever P, Macrae VE MiRNA-221 and miRNA-222 synergistically function to promote vascular calcification. Cell Biochem Funct 2014; 32(2): 209-16.

[42] Gui B, Hsieh CL, Kantoff PW, Kibel AS, Jia L. Androgen receptor-mediated downregulation of microRNA-221 and -222 in castration-resistant prostate cancer. PLoS One 2017; 12(9): e0184166.

[43] Liu W, Ling S, Sun W, et al. Circulating microRNAs correlated with the level of coronary artery calcification in symptomatic patients. Sci Rep 2015; 5: 16099.

[44] Friberg L, Benson L, Rosenqvist M, Lip GY. Assessment of female sex as a risk factor in atrial fibrillation in Sweden: nationwide retrospective cohort study. BMJ 2012; 344: e3522.

[45] Weijs B, Blaauw Y, Rennenberg RJ, et al. Patients using vitamin K antagonists show increased levels of coronary calcification: an observational study in low-risk atrial fibrillation patients. Eur Heart J 2011; 32(20): 2555-62. 